\title{
Valley Hall effect and nonlocal resistance in locally gapped graphene
}

\author{
Aktor, Thomas; Garcia, Jose H.; Roche, Stephan; Jauho, Antti Pekka; Power, Stephen R.
}

Published in:

Physical Review B

Link to article, DOI:

10.1103/PhysRevB.103.115406

Publication date:

2021

Document Version

Publisher's PDF, also known as Version of record

Link back to DTU Orbit

Citation (APA):

Aktor, T., Garcia, J. H., Roche, S., Jauho, A. P., \& Power, S. R. (2021). Valley Hall effect and nonlocal resistance in locally gapped graphene. Physical Review B, 103(11), [115406].

https://doi.org/10.1103/PhysRevB.103.115406

\section{General rights}

Copyright and moral rights for the publications made accessible in the public portal are retained by the authors and/or other copyright owners and it is a condition of accessing publications that users recognise and abide by the legal requirements associated with these rights.

- Users may download and print one copy of any publication from the public portal for the purpose of private study or research.

- You may not further distribute the material or use it for any profit-making activity or commercial gain

- You may freely distribute the URL identifying the publication in the public portal

If you believe that this document breaches copyright please contact us providing details, and we will remove access to the work immediately and investigate your claim 


\title{
Valley Hall effect and nonlocal resistance in locally gapped graphene
}

\author{
Thomas Aktor $\odot,{ }^{1}$ Jose H. Garcia, ${ }^{2}$ Stephan Roche, ${ }^{2,3}$ Antti-Pekka Jauho, ${ }^{1}$ and Stephen R. Power ${ }^{4, *}$ \\ ${ }^{1}$ Center for Nanostructured Graphene, DTU Physics, Technical University of Denmark, DK-2800 Kongens Lyngby, Denmark \\ ${ }^{2}$ Catalan Institute of Nanoscience and Nanotechnology, CSIC and The Barcelona Institute of Science and Technology, \\ Campus UAB, Bellaterra, 08193 Barcelona (Cerdanyola del Vallès), Spain \\ ${ }^{3}$ Institució Catalana de Recerca i Estudis Avançats, 08070 Barcelona, Spain \\ ${ }^{4}$ School of Physics, Trinity College Dublin, Dublin 2, Ireland
}

(Received 18 September 2019; accepted 18 February 2021; published 4 March 2021)

\begin{abstract}
We report on the emergence of bulk, valley-polarized currents in graphene-based devices, driven by spatially varying regions of broken sublattice symmetry, and revealed by nonlocal resistance $\left(R_{\mathrm{NL}}\right)$ fingerprints. By using a combination of quantum transport formalisms, giving access to bulk properties as well as multiterminal device responses, the presence of a nonuniform local band gap is shown to give rise to valley-dependent scattering and a finite Fermi-surface contribution to the valley Hall conductivity, related to characteristics of $R_{\mathrm{NL}}$. These features are robust against disorder and provide a plausible interpretation of controversial experiments in graphene/hexagonal boron nitride superlattices. Our findings suggest both an alternative mechanism for the generation of valley Hall effect in graphene and a route towards valley-dependent electron optics, by materials and device engineering.
\end{abstract}

DOI: 10.1103/PhysRevB.103.115406

Two-dimensional materials, and graphene in particular, are promising valleytronic candidates due to the $K$ and $K^{\prime}$ valleys at the Dirac points, which can be exploited to encode, transport, and process information [1] and could play a key role in future valley-driven quantum computers [2-4]. However, a key hurdle is the absence of external knobs, analogous to magnetic fields and ferromagnetic contacts in spintronics, to generate, manipulate, and detect valley-polarized currents [5]. While optoelectronic access to the valley index is possible in certain materials [6-9], an all-electronic control would be highly desirable for device applications [10]. Certain defects or strain deformation fields have been theoretically proposed for achieving valley filtering, but the corresponding experimental implementation remains challenging [11-33]. Possible signatures of valley transport phenomena have been discussed in relation to nonlocal resistance $\left(R_{\mathrm{NL}}\right)$ measurements in commensurately stacked graphene/hexagonal boron nitride (hBN) systems [34-36]. Large $R_{\mathrm{NL}}$ signals have been interpreted in terms of an intrinsic valley Hall effect (VHE), driven by bulk Berry curvature [37-40], and which would be related to a uniform mass term induced by the interaction between graphene and hBN. Under this mechanism, a quantized valley Hall conductivity, $\sigma_{x y}^{v}=\frac{2 e^{2}}{h}$, within the band gap [5,34,39,41], was argued to generate a long-ranged valley current, enhancing $R_{\mathrm{NL}}$ beyond standard ohmic contributions. Analogous behavior has also been studied in bilayer graphene, where the VHE is tunable by an interlayer bias $[42,43]$.

However, the interpretation of experimental $R_{\mathrm{NL}}$ in terms of a bulk-driven VHE has been severely questioned by quantum transport simulations $[5,44,45]$. Bulk-driven $R_{\mathrm{NL}}$ signals

\footnotetext{
*stephen.power@tcd.ie
}

in fully gapped systems are found to be strongly suppressed beyond evanescent contributions, rendering them fully negligible at experimental scales. One puzzling issue is that intrinsic valley Hall currents are associated with Fermi sea contributions from Berry curvature hotspots below the Fermi energy, whereas within the relevant linear response regime only Fermi-surface (FS) contributions should play a role in device measurements $[44,45]$. Experimental mapping of current flow further suggested that edge currents may play a role [46], but recent theoretical $[45,47,48]$ and experimental [49] studies cast doubt upon the topological origin of such currents. Finally, the lattice mismatch between graphene and $\mathrm{hBN}$, which leads to a moiré pattern for commensurate structures [34,50], also clearly indicates that electrons may not experience a uniform mass term [51], making the interpretation of experimental $R_{\mathrm{NL}}$ signals in terms of VHE a true and unsolved conundrum.

In this paper, we demonstrate the emergence of valleysplit bulk transport in the absence of a global band gap. This phenomenon requires instead the presence of a spatially varying mass term-a situation analogous to graphene-hBN heterostructures with commensurate layer alignment. The valley-polarized current is a consequence of an extrinsic-like valley Hall effect that can be understood by examining the scattering from a single, circular mass dot. The exact solution to this case establishes a strong valley dependence in the scattered wave function at low energies and a valley splitting of incoming electronic currents. Tight-binding simulations show that this effect is robust for various dot profiles and mass distributions. The valley Hall conductivity for a periodic array of dots is then calculated using the Kubo-Bastin formalism, and confirms the formation of valley Hall signals and charge neutral currents. This system 
(a)

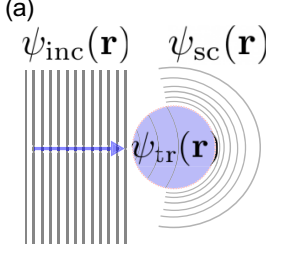

(b)

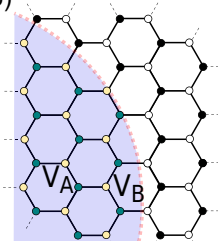

(d)

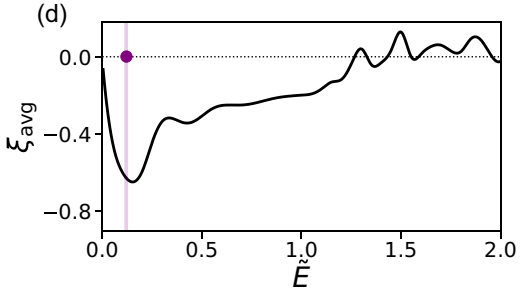

(c)
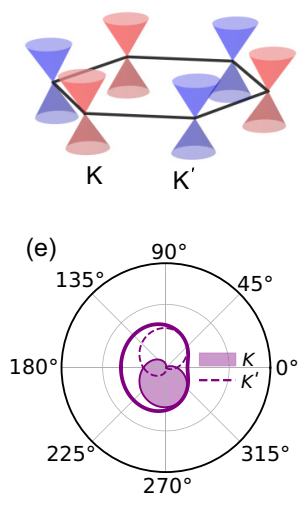

FIG. 1. (a) Incoming, scattered, and transmitted waves for scattering from a mass dot. (b) $A$ and $B$ sublattice sites in the dot have different onsite potentials. (c) $K$ and $K^{\prime}$ valleys in the low-energy spectrum of graphene. (d) Valley polarization of scattered currents: the peak indicates a strong $K^{\prime}$ polarization in the $+y$ direction. (e) Total (bold) and individual valley angular scattering profiles in the far-field limit at $\tilde{E}=0.12$ [purple dot in (d)].

manifests a nonzero FS contribution to the valley Hall conductivity, consistent with the extrinsic contribution and in contrast to the spatially uniform band-gap case [41]. Using Landauer-Buttiker simulations, we show that scatteringinduced valley-polarized currents significantly enhance $R_{\mathrm{NL}}$ signals, in contrast to the vanishing $R_{\mathrm{NL}}$ predicted for uniform mass systems. Scattering-induced valley splitting, unlike Berry-phase induced deflection, is consistent with standard quantum transport methods, and provides an alternative mechanism to interpret valleytronic phenomena using $R_{\mathrm{NL}}$ measurements. It also suggests a route towards valley engineering by using hBN or other substrates [50-53], patterned gates [54], or doping [55-62] to generate spatially varying mass profiles in graphene to direct the flow of valley currents.

Scattering from a circular mass dot is considered using a Dirac Hamiltonian near the $K$ and $K^{\prime}(\tau= \pm 1)$ points:

$$
\mathcal{H}_{\tau}(\mathbf{r})=\hbar v_{F}\left(\begin{array}{cc}
\tilde{V}_{A}(\mathbf{r}) & -i \tau \partial_{x}-\partial_{y} \\
-i \tau \partial_{x}+\partial_{y} & \tilde{V}_{B}(\mathbf{r})
\end{array}\right),
$$

with scaled variables $\tilde{X} \equiv \frac{X}{\hbar v_{F}}$. Here $V_{A}(\mathbf{r})$ and $V_{B}(\mathbf{r})$ are the $A$ and $B$ sublattice potentials. Within a dot of radius $R$ we set $V_{A / B}(r<R)= \pm \frac{\Delta}{2}$, where the sign depends on the sublattice. The mass term $\Delta$ leads to band-gap opening in the range $-\frac{\Delta}{2}<E<\frac{\Delta}{2}$ in the region where $V_{A / B} \neq 0$. Following Refs. [63-65], we switch to polar coordinates $(r, \phi)$ and consider incoming $\left(\psi_{\text {inc }}\right)$, scattered $\left(\psi_{\mathrm{sc}}\right)$, and transmitted $\left(\psi_{\text {tr }}\right)$ waves, as shown in Fig. 1(a). $\psi_{\text {inc }}$ and $\psi_{\text {sc }}$ are expanded in terms of angular momentum basis states $m$ using Bessel and Hankel functions, respectively, as in the potential dot case $[64,65]$. Inside the dot, $\psi_{\text {tr }}$ is expanded in terms of Bessel functions with sublattice-dependent coefficients (see Supplemental Material [66]). A closed-form expression for the wave function is found by enforcing continuity at the dot boundary and solving for the scattering and transmission coefficients, $c_{m}^{s}$ and $c_{m}^{t}$, for each mode. The Hamiltonian in Eq. (1) is

invariant under $V_{A} \leftrightarrow V_{B}, \psi_{1}^{\tau} \leftrightarrow \psi_{2}^{-\tau}, \psi_{2}^{\tau} \leftrightarrow-\psi_{1}^{-\tau}$, so that the $K$ valley result can be used to deduce the $K^{\prime}$ case. The local electron density $n=\psi^{\dagger} \psi$ and particle current $\mathbf{j}=\psi^{\dagger} \sigma \psi$ are calculated for each valley separately, with the total electronic (valley) quantity given by the sum (difference) of the two valleys. The local valley polarization at a point $(r, \phi)$ is the ratio of these quantities $\xi(r, \phi) \equiv j_{\text {val }}^{\mathrm{sc}}(r, \phi) / j_{\text {tot }}^{\mathrm{sc}}(r, \phi)$, and takes values $-1,0,1$ for fully $K^{\prime}$ polarized, valley neutral, and fully $K$ polarized currents, respectively. A figure of merit for the valley-splitting efficiency of a dot is

$$
\xi_{\text {avg }}=\lim _{r \rightarrow \infty} \int_{0}^{\pi} \frac{d \phi}{\pi} \xi^{\mathrm{sc}}(r, \phi),
$$

i.e., the far-field limit of the scattered current polarization, averaged over the upper-half plane. This quantifies the average valley polarization of transverse currents, with nonzero $\xi_{\text {avg }}$ indicating VHE-type behavior. We consider a $R=4.5$ dot with $\tilde{\Delta}=2$ so that the band edge inside the dot is at $\tilde{E}=$ \pm 1 , and focus here on valley-dependent effects-for general scattering properties see [66]. In Fig. 1(d), $\xi_{\text {avg }}$ takes large negative values as $E$ increases from zero, before decaying to about half its maximum magnitude, and then vanishing at energies above the band edge. This corresponds to $K^{\prime}$ electrons preferentially scattering in the $+y$ direction for all band-gap energies, with the strongest effect near the gap center. The angular dependence of scattering is determined from the radial component of $\mathbf{j}^{\text {sc }}(r \rightarrow \infty)$. Figure 1(e) plots the total quantity (solid line) and its individual valley contributions (shaded and unshaded areas) at the energy shown by the dot in Fig. 1(d). $K$ and $K^{\prime}$ have equal contributions at $\phi=0, \pi$, corresponding to a valley-neutral situation for exact forward and back scattering from the dot. At other angles the two valleys scatter antisymmetrically with respect to the $x$ axis. At the energy shown, scattering is largest in the transverse directions so that electrons from different valleys scatter almost entirely to opposite sides of the dot.

Figures 2(a)-2(c) show the current flow near the dot at this energy, with arrows showing the current magnitude and direction in each case. Figure 2(a) shows the total charge flow, with the color scale showing valley polarization, whereas Figs. 2(b) and 2(c) show the current flow and electron density for each valley separately. $K$ and $K^{\prime}$ electrons flow in opposite directions around the dot, leading to the same antisymmetry around the $x$ axis that was noted for angular scattering. Valley-polarized currents are observed in the immediate vicinity of the dot in Fig. 2(a), but quickly recombine to give a largely valley-neutral forward current. This is also evident from Figs. 2(b) and 2(c), where the large $K\left(K^{\prime}\right)$ current near the top (bottom) of the dot diffuses quickly behind it. The far-field behavior emerges from currents further away, namely, the $K\left(K^{\prime}\right)$ current flowing in the bottom (top) left of these panels.

Counterpropagating flows of $K$ and $K^{\prime}$ currents emerge from the scattering coefficients $c_{\bar{m}}^{s}(K)$ of the individual modes, the analytic form of which is given in [66]. The $m$ and $\bar{m} \equiv-m-1$ modes are closely connected, and we find $c_{\bar{m}}^{s}(K)=c_{m}^{s}\left(K^{\prime}\right)$, so that every $K$ valley mode has a corresponding $K^{\prime}$ mode which contributes with equal magnitude but opposite angular momentum. At low energies, only $m, \bar{m}=0$ modes contribute, and the clearest valley splitting 

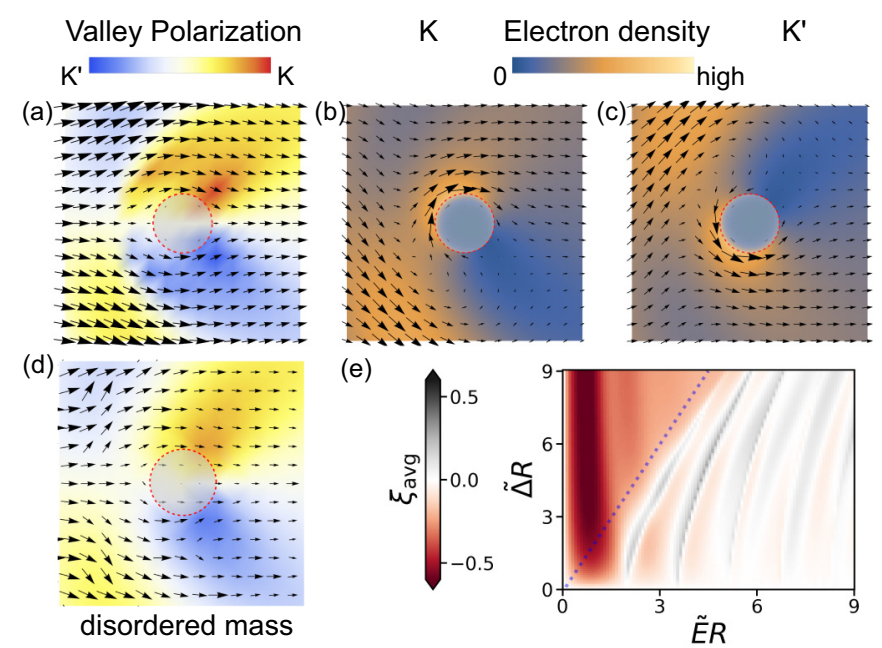

(e)

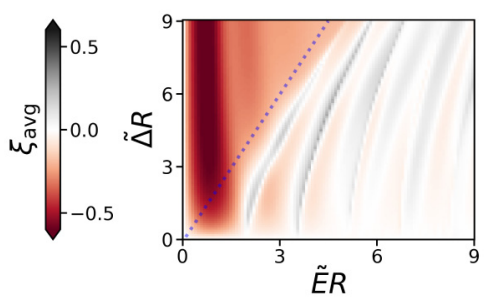

FIG. 2. (a) Total and (b), (c) individual valley current flows near a mass dot at low energy. Electrons from different valleys flow in opposite directions around the dot. (d) Similar valley-splitting behavior for a disordered mass region. (e) Phase space map for the average valley polarization $\xi_{\text {avg }}$ for different $\tilde{E}$ and $\tilde{\Delta}$ values, with the dotted line denoting the band edge.

is observed. As $E$ increases, there is a gradual onset of contributions from $m, \bar{m}>0$ modes, and valley-splitting effects from different modes partially cancel, leading to a decrease in the magnitude of $\xi_{\text {avg }}$ [Fig. 1(d)]. However, the presence of higher-order modes maintains the sign of $\xi_{\text {avg }}$ within the gap, so that the deflection direction is consistent. This is clear from Fig. 2(e), where we show $\xi_{\text {avg }}$ across a range of mass strengths and electron energies. The dotted line denotes the band edge, and a uniform preference for enhanced $K^{\prime}$ scattering is seen inside the gap, with a weak oscillatory pattern outside due to resonances with bound states. The strongest valley polarization (dark red region) is at low energies for all sizes and strengths. For further discussion of the energy dependence, see [66].

To test the robustness of the valley splitting predicted above, we perform tight-binding simulations using the patched Green's-function approach [24,67]. A finite dot with $R=10 \mathrm{~nm}$ and $V_{A / B}(r<R)= \pm 0.1|t|$ is embedded in an infinite graphene sheet, and we perform transport calculations using a point probe $250 \mathrm{~nm}$ away so that incoming electrons resemble a plane wave, as in Fig. 1(a). The system Green's functions [68], $G(E)$, and lead broadening term, $\Gamma$, give the spectral density of injected states $A(E)=$ $G \Gamma G^{+}$and local current flow in real space. Projecting $A$ onto the graphene basis $\left|\psi_{k}\right\rangle$ measures the local distribution of scattered states in $k$ space, $\rho(\boldsymbol{k})=\left\langle\psi_{k}|A| \psi_{k}\right\rangle$, from which the real-space valley polarization is calculated: $\xi_{T B}=$ $\left(\sum_{\boldsymbol{k} \in K} \rho(\boldsymbol{k})-\sum_{\boldsymbol{k} \in K^{\prime}} \rho(\boldsymbol{k})\right) / \sum_{\boldsymbol{k} \in K, K^{\prime}} \rho(\boldsymbol{k})$. The analytic polarization from Fig. 2(a) is reproduced numerically [66] not only for a perfect dot, but also for nonuniform mass distributions. Figure 2(d) shows a disordered case, where $V_{A / B} \neq 0$ only on $1 \%$ of sites within the dot, but with increased magnitudes to preserve the average mass. The result, for this and other mass distributions, is almost identical to the analytic, perfect dot prediction. Insensitivity to the strength, size, or
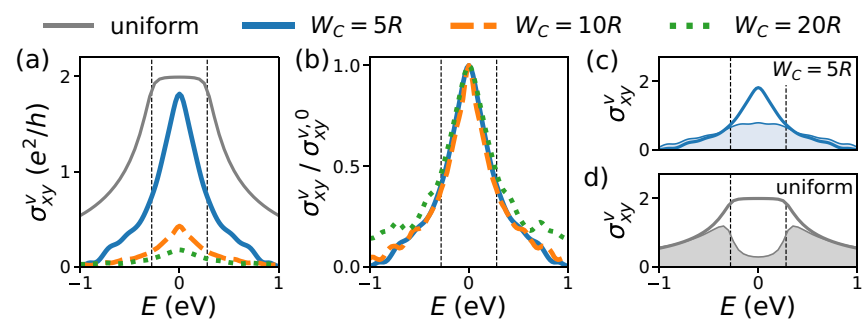

FIG. 3. (a) Valley Hall conductivity $\sigma_{x y}^{v}$, and (b) $\sigma_{x y}^{v}$ normalized by its peak value, for three different supercell sizes. (c), (d) $\sigma_{x y}^{v}$ for $\mathrm{W}_{C}=5 \mathrm{R}$ and uniform mass distributions, together with their Fermisurface contribution (shaded). Dashed vertical lines show the band edges in mass regions.

composition of mass regions suggests that realization of valleytronic devices may be easier to achieve using mass dot engineering than strain-based proposals.

Mass dots induce equal and opposite deflections of $K$ and $K^{\prime}$ electrons in the $y$ direction, giving rise to a pure valley current in the transverse direction, which is reminiscent of Berry curvature deflections in globally gapped systems $[5,34,39,41]$. To determine possible experimental signatures of this phenomenon, we analyze the valley Hall conductivity $\sigma_{x y}^{v}$ and nonlocal resistance $R_{\mathrm{NL}}$ using both the Kubo and Landauer-Buttiker formalisms. We employ the Kubo-Bastin formula [26,69-76] to calculate both the full and Fermi-surface contributions to $\sigma_{x y}^{v}$ [66] for a $R=10 \mathrm{~nm}$ mass dot $\left(V_{A / B}= \pm 0.1|t|\right)$ placed into one of three different square supercells with side lengths $W_{C}=5 R, 10 R, 20 R$. This allows us to examine the competition between scattering and Berry curvature effects, which can arise in periodic systems due to a finite concentration $c$ of sites with mass $\Delta$, yielding an effective global mass $\approx c \Delta$. For a global mass, the height of the $\sigma_{x y}^{v}$ peak remains constant, with wider peaks expected for larger $\Delta_{\text {eff }}$ (smaller $W_{C}$ ). However, Fig. 3(a) shows that instead the height decreases with decreasing dot density, while the width is independent of $W_{C}$, so that the three curves coincide when normalized [Fig. 3(b)]. This suggests a valley-splitting mechanism analogous to an extrinsic spin Hall effect induced by skew scattering $[77,78]$. In this case, the magnitude of $\sigma_{x y}^{v}$ should vary with the dot density, but with an energy dependence following the scattering profile of a single dot, as observed. To further substantiate this hypothesis, we examine the FS contributions [72,79] to $\sigma_{x y}^{v}$, which remain finite throughout the band gap for the mass dot system in Fig. 3(c), with $\sigma_{x y}^{v, \mathrm{FS}} \approx 0.4 \sigma_{x y}^{v}$ at $E=0$. For a uniform mass [Fig. 3(d)], this contribution vanishes (aside from broadening effects [66]) in the gap. Similar behavior is found for each $W_{C}$, demonstrating that a robust FS contribution emerges in the presence of spatially distributed mass dots.

The absence of a global gap enables straightforward $R_{\mathrm{NL}}$ simulations using Landauer-Buttiker methods. We consider the six-terminal setup in Fig. 4(a), where $R=5 \mathrm{~nm}$ $\operatorname{dots}\left(V_{A / B}= \pm 0.4|t|\right)$ with periodicity $W_{C} \sim 6 R$ are embedded in a 64-nm-wide zigzag ribbon. The currents $I_{p}$ and potentials $V_{p}$ in each lead $p$ are related by $I_{p}=$ $\frac{2 e}{h} \sum\left(T_{q p} V_{p}-T_{p q} V_{q}\right)$, where transmissions $T_{p q}$ are calculated using recursive Green's-function techniques [67,80,81]. The 


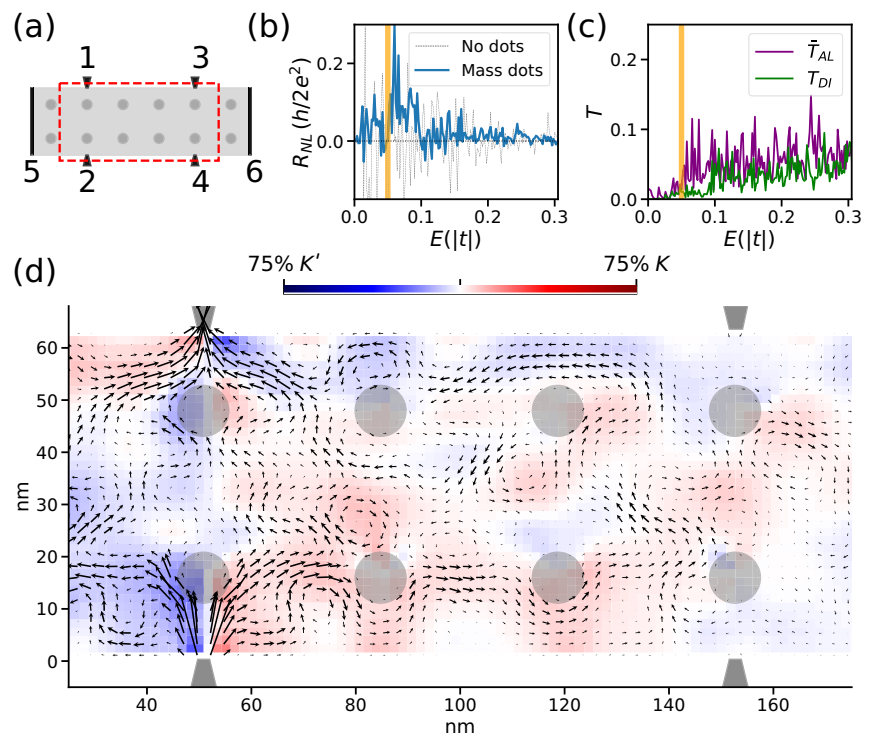

FIG. 4. (a) Six-terminal device for $R_{\mathrm{NL}}$ simulations. (b) $\mathrm{R}_{\mathrm{NL}}$ with and without mass dots, showing a robust positive trend in the presence of dots. (c) Transmission along one edge $\left(\mathrm{T}_{A L}\right)$ and diagonally across the device $\left(\mathrm{T}_{D I}\right)$. (d) Map of local current flow (arrows) and valley polarization (color) for the energy shown in orange in (b) and (c). $\mathrm{R}_{\mathrm{NL}}$ is mediated by valley-polarized currents in the device.

potential difference between leads 1 and 2 is fixed, and the net current in the remaining leads set to zero. Solving for the current, $I_{1}=-I_{2}$, and the potentials $V_{3-6}$ yields $R_{\mathrm{NL}}=\frac{V_{3}-V_{4}}{I}$. In the absence of dots, $R_{\mathrm{NL}}$ oscillates rapidly around zero, as shown by the dashed line in Fig. 4(b). These oscillations are due to the finite device size, and emerge from configurationspecific resonances in the pairwise transmissions $T_{p q}$. Unlike $\sigma_{x y}^{v}$, the $R_{\mathrm{NL}}$ signal is not mediated solely by Hall effects, but is sensitive to the device size, edge types, probe placements, and the distances between scatterers and edges. Such effects are difficult to remove from the simulations, but average out in experimental-scale systems to give a vanishing contribution to the $R_{\mathrm{NL}}$ signal. This represents the current taking a direct path between source and drain, and not probing the nonlocal region of the device. With mass dots present (solid blue curve), $R_{\mathrm{NL}}$ still displays rapid oscillations due to finite-size effects, but a wide positive peak feature now emerges at low energies. Similar behavior is seen for different dot sizes and separations, mass strengths, and disorder types, but does not occur in pristine systems or for dots with $V_{A}=V_{B}$.

To understand the origin of the positive $R_{\mathrm{NL}}$ signal, we analyze the pairwise transmission profiles between probes. The sign of $R_{\mathrm{NL}}$ [66] is a competition between transmissions along an edge of the device $\left(T_{A L}\right)$ and diagonally across the device $\left(T_{D I}\right)$. Figure 4 (c) confirms that the positive $R_{\mathrm{NL}}$ feature corresponds to an enhancement of $T_{A L}$ relative to $T_{D I}$ for our system. To better interpret the dominant $R_{\mathrm{NL}}$ signal, we map the local current throughout the device. In multiterminal devices, the current flow is not simply an injection at the source and absorption at the drain. It results from a superposition of currents injected and absorbed by each probe [81-85]. The arrows in Fig. 4(d) show the current in our system for the energy marked by orange lines in Figs. 4(b) and 4(c). Due to finite-size effects, the flow pattern is complex and sensitive to the chosen energy. Streams and vortices appear, disappear, and relocate as the energy is varied. However, at low energies, strong transverse scattering from the dots leads to indirect current paths which probe large portions of the device. This is in stark contrast to pristine systems, where more direct paths between source and drain are observed [66]. The valley polarization of the current, calculated in the multiterminal setup, is shown here by color shading. We note that strong valley splitting at dots near the source and drain contacts, reinforced by subsequent scattering from other dots, leads to valley-polarized currents throughout the entire device.

Electrons from the two valleys tend to flow in opposite directions near the edge; for example, near the bottom edge, $K$ $\left(K^{\prime}\right)$ currents flow primarily to the right (left). This may appear analogous to the quantum spin Hall effect, where electrons of different spin counterpropagate along an edge, but there are fundamental differences. First the studied system does not have an insulating bulk, but rather strong transverse scattering sources which deflect valley-polarized current towards the device edges. An important aspect here is that significant current still flows in the regions between dots in the bulk and valleypolarized currents near the edge are not protected or carried by chiral states: they arise due to scattering effects and can be quenched, be deflected, or change polarization. However, cumulative scattering from consecutive dots generally acts to reinforce valley polarization, and to boost $T_{A L}$ by deflecting current along the edge. This leads to the positive $R_{\mathrm{NL}}$ feature seen in Fig. 4(b), with similar results found for different device sizes, dot densities, ribbon edges, and disorders. $R_{\mathrm{NL}}$ signals, mediated by scattering-induced valley splitting, are a general feature of graphene with nonuniform mass distributions.

We calculated the electron scattering from mass dots in graphene, and obtained a clear splitting of electrons according to their valley index. This effect is robust over a wide range of dot sizes, mass distributions, and electron energies. A nonuniform mass distribution, consisting of an array of such dots, gives rise to a valley Hall conductivity which contrasts sharply with that of the uniform mass case and displays a significant Fermi-surface contribution. Furthermore, such arrays give rise to a positive $R_{\mathrm{NL}}$ feature in Hall bar devices, without requiring Berry curvature driven, Fermi sea currents in the system bulk. Instead, $R_{\mathrm{NL}}$ signals are driven by cumulative scattering which generates bulk, Fermi-surface, valley-polarized currents and enhances transmission along the device edges. While the complicated mass profile in graphene/hBN heterostructures [51] makes it difficult to disentangle the different mechanisms at play in this system, nonuniform mass distributions are an ideal platform to further clarify the role of valley currents in the emergence of nonzero $R_{\mathrm{NL}}$ signals. Our results suggest mass-based nanostructures are a robust alternative to strained systems to achieve novel valley-dependent electron optics.

S.R.P. acknowledges funding from the Irish Research Council under the Laureate awards program. J.H.G. and S.R. acknowledge funding from the European Union Seventh Framework Programme under Grant No. 881603 (Graphene Flagship). ICN2 is funded by the CERCA Programme/Generalitat de Catalunya and supported by the 
Severo Ochoa programme (MINECO Grant. No. SEV-20170706). The Center for Nanostructured Graphene is sponsored by the Danish National Research Foundation, Project No. DNRF103.
[1] J. R. Schaibley, H. Yu, G. Clark, P. Rivera, J. S. Ross, K. L. Seyler, W. Yao, and X. Xu, Nat. Rev. Mater. 1, 16055 (2016).

[2] N. Rohling and G. Burkard, New J. Phys. 14, 083008 (2012).

[3] D. Culcer, A. L. Saraiva, B. Koiller, X. Hu, and S. Das Sarma, Phys. Rev. Lett. 108, 126804 (2012).

[4] E. A. Laird, F. Pei, and L. P. Kouwenhoven, Nat. Nanotechnol. 8, 565 (2013).

[5] A. Cresti, B. K. Nikolić, J. H. García, and S. Roche, Riv. del Nuovo Cim. 39, 587 (2016).

[6] D. Xiao, G.-B. Liu, W. Feng, X. Xu, and W. Yao, Phys. Rev. Lett. 108, 196802 (2012).

[7] T. Cao, G. Wang, W. Han, H. Ye, C. Zhu, J. Shi, Q. Niu, P. Tan, E. Wang, B. Liu et al., Nat. Commun. 3, 887 (2012).

[8] Y. Li, J. Ludwig, T. Low, A. Chernikov, X. Cui, G. Arefe, Y. D. Kim, A. M. van der Zande, A. Rigosi, H. M. Hill, S. H. Kim, J. Hone, Z. Li, D. Smirnov, and T. F. Heinz, Phys. Rev. Lett. 113, 266804 (2014).

[9] Z. Zhang, X. Ni, H. Huang, L. Hu, and F. Liu, Phys. Rev. B 99, 115441 (2019).

[10] Y. S. Ang, S. A. Yang, C. Zhang, Z. Ma, and L. K. Ang, Phys. Rev. B 96, 245410 (2017).

[11] A. Rycerz, J. Tworzydło, and C. Beenakker, Nat. Phys. 3, 172 (2007).

[12] J. L. Garcia-Pomar, A. Cortijo, and M. Nieto-Vesperinas, Phys. Rev. Lett. 100, 236801 (2008).

[13] T. Fujita, M. Jalil, and S. Tan, Appl. Phys. Lett. 97, 043508 (2010)

[14] D. Gunlycke and C. T. White, Phys. Rev. Lett. 106, 136806 (2011).

[15] J.-H. Chen, G. Autès, N. Alem, F. Gargiulo, A. Gautam, M. Linck, C. Kisielowski, O. V. Yazyev, S. G. Louie, and A. Zettl, Phys. Rev. B 89, 121407(R) (2014).

[16] M. M. Asmar and S. E. Ulloa, Phys. Rev. B 96, 201407(R) (2017).

[17] C. Park, Phys. Rev. Applied 11, 044033 (2019).

[18] N. Levy, S. Burke, K. Meaker, M. Panlasigui, A. Zettl, F. Guinea, A. H. Castro Neto, and M. Crommie, Science 329, 544 (2010).

[19] F. Guinea, B. Horovitz, and P. Le Doussal, Phys. Rev. B 77, 205421 (2008).

[20] F. Guinea, M. Katsnelson, and A. Geim, Nat. Phys. 6, 30 (2010).

[21] M. Vozmediano, M. Katsnelson, and F. Guinea, Phys. Rep. 496, 109 (2010).

[22] M. Settnes, S. R. Power, and A.-P. Jauho, Phys. Rev. B 93, 035456 (2016).

[23] Z. Qi, D. Bahamon, V. M. Pereira, H. S. Park, D. Campbell, and A. H. Castro Neto, Nano Lett. 13, 2692 (2013).

[24] M. Settnes, S. R. Power, M. Brandbyge, and A.-P. Jauho, Phys. Rev. Lett. 117, 276801 (2016).

[25] S. Milovanović and F. Peeters, Appl. Phys. Lett. 109, 203108 (2016).

[26] M. Settnes, J. H. Garcia, and S. Roche, 2D Mater. 4, 031006 (2017).

[27] D. Zhai and N. Sandler, Phys. Rev. B 98, 165437 (2018).
[28] T. Stegmann and N. Szpak, 2D Mater. 6, 015024 (2018).

[29] Y. Wu, D. Zhai, C. Pan, B. Cheng, T. Taniguchi, K. Watanabe, N. Sandler, and M. Bockrath, Nano Lett. 18, 64 (2018).

[30] E. Andrade, R. Carrillo-Bastos, and G. G. Naumis, Phys. Rev. B 99, 035411 (2019).

[31] T. Kariyado, J. Phys. Soc. Jpn. 88, 083701 (2019).

[32] A. C. McRae, G. Wei, and A. R. Champagne, Phys. Rev. Applied 11, 054019 (2019).

[33] V. Torres, P. Silva, E. A. T. de Souza, L. A. Silva, and D. A. Bahamon, Phys. Rev. B 100, 205411 (2019).

[34] R. Gorbachev, J. Song, G. Yu, A. Kretinin, F. Withers, Y. Cao, A. Mishchenko, I. Grigorieva, K. Novoselov, L. Levitov et al., Science 346, 448 (2014).

[35] K. Komatsu, Y. Morita, E. Watanabe, D. Tsuya, K. Watanabe, T. Taniguchi, and S. Moriyama, Sci. Adv. 4, eaaq0194 (2018).

[36] K. Endo, K. Komatsu, T. Iwasaki, E. Watanabe, D. Tsuya, K. Watanabe, T. Taniguchi, Y. Noguchi, Y. Wakayama, Y. Morita et al., Appl. Phys. Lett. 114, 243105 (2019).

[37] Y. D. Lensky, J. C. W. Song, P. Samutpraphoot, and L. S. Levitov, Phys. Rev. Lett. 114, 256601 (2015).

[38] D. Xiao, W. Yao, and Q. Niu, Phys. Rev. Lett. 99, 236809 (2007).

[39] T. Ando, J. Phys. Soc. Jpn. 84, 114705 (2015).

[40] M. Beconcini, F. Taddei, and M. Polini, Phys. Rev. B 94, 121408(R) (2016).

[41] J. C. W. Song, P. Samutpraphoot, and L. S. Levitov, Proc. Natl. Acad. Sci. USA 112, 10879 (2015).

[42] M. Sui, G. Chen, L. Ma, W.-Y. Shan, D. Tian, K. Watanabe, T. Taniguchi, X. Jin, W. Yao, D. Xiao et al., Nat. Phys. 11, 1027 (2015).

[43] Y. Shimazaki, M. Yamamoto, I. V. Borzenets, K. Watanabe, T. Taniguchi, and S. Tarucha, Nat. Phys. 11, 1032 (2015).

[44] G. Kirczenow, Phys. Rev. B 92, 125425 (2015).

[45] J. M. Marmolejo-Tejada, J. H. García, M. D. Petrović, P.-H. Chang, X.-L. Sheng, A. Cresti, P. Plecháč, S. Roche, and B. K. Nikolić, J. Phys. Mater. 1, 015006 (2018).

[46] M. Zhu, A. Kretinin, M. D. Thompson, D. Bandurin, S. Hu, G. Yu, J. Birkbeck, A. Mishchenko, I. Vera-Marun, K. Watanabe et al., Nat. Commun. 8, 14552 (2017).

[47] R. Brown, N. R. Walet, and F. Guinea, Phys. Rev. Lett. 120, 026802 (2018).

[48] J. C. W. Song and G. Vignale, Phys. Rev. B 99, 235405 (2019).

[49] A. Aharon-Steinberg, A. Marguerite, D. J. Perello, K. Bagani, T. Holder, Y. Myasoedov, L. S. Levitov, A. K. Geim, and E. Zeldov, arXiv:2012.02842 (2020).

[50] C. Woods, L. Britnell, A. Eckmann, R. Ma, J. Lu, H. Guo, X. Lin, G. Yu, Y. Cao, R. Gorbachev et al., Nat. Phys. 10, 451 (2014).

[51] J. Jung, A. M. DaSilva, A. H. MacDonald, and S. Adam, Nat. Commun. 6, 6308 (2015).

[52] H. X. Yang, A. Hallal, D. Terrade, X. Waintal, S. Roche, and M. Chshiev, Phys. Rev. Lett. 110, 046603 (2013). 
[53] C. Forsythe, X. Zhou, K. Watanabe, T. Taniguchi, A. Pasupathy, P. Moon, M. Koshino, P. Kim, and C. R. Dean, Nat. Nanotechnol. 13, 566 (2018).

[54] R. Huber, M.-H. Liu, S.-C. Chen, M. Drienovsky, A. Sandner, K. Watanabe, T. Taniguchi, K. Richter, D. Weiss, and J. Eroms, Nano Lett. 20, 8046 (2020).

[55] L. Zhao, R. He, K. T. Rim, T. Schiros, K. S. Kim, H. Zhou, C. Gutiérrez, S. Chockalingam, C. J. Arguello, L. Pálová et al., Science 333, 999 (2011).

[56] R. Lv, Q. Li, A. R. Botello-Méndez, T. Hayashi, B. Wang, A. Berkdemir, Q. Hao, A. L. Elías, R. Cruz-Silva, H. R. Gutiérrez et al., Sci. Rep. 2, 586 (2012).

[57] D. Y. Usachov, A. V. Fedorov, O. Y. Vilkov, A. E. Petukhov, A. G. Rybkin, A. Ernst, M. M. Otrokov, E. V. Chulkov, I. I. Ogorodnikov, M. V. Kuznetsov et al., Nano Lett. 16, 4535 (2016).

[58] A. Zabet-Khosousi, L. Zhao, L. Pálová, M. S. Hybertsen, D. R. Reichman, A. N. Pasupathy, and G. W. Flynn, J. Am. Chem. Soc. 136, 1391 (2014).

[59] J. A. Lawlor and M. S. Ferreira, Beils. J. Nanotechnol. 5, 1210 (2014).

[60] J. A. Lawlor, P. D. Gorman, S. R. Power, C. G. Bezerra, and M. S. Ferreira, Carbon 77, 645 (2014).

[61] A. Lherbier, A. R. Botello-Mendez, and J.-C. Charlier, Nano Lett. 13, 1446 (2013).

[62] T. Aktor, A.-P. Jauho, and S. R. Power, Phys. Rev. B 93, 035446 (2016).

[63] A. Ferreira, J. Viana-Gomes, J. Nilsson, E. R. Mucciolo, N. M. R. Peres, and A. H. Castro Neto, Phys. Rev. B 83, 165402 (2011).

[64] R. L. Heinisch, F. X. Bronold, and H. Fehske, Phys. Rev. B 87, 155409 (2013).

[65] C. Schulz, R. L. Heinisch, and H. Fehske, Quantum Matter 4, 346 (2015).

[66] See Supplemental Material at http://link.aps.org/supplemental/ 10.1103/PhysRevB.103.115406 for the analytic solution to the scattering problem, a discussion of mode coefficients, and details of the Kubo-Bastin calculations.

[67] M. Settnes, S. R. Power, J. Lin, D. H. Petersen, and A.-P. Jauho, Phys. Rev. B 91, 125408 (2015).

[68] S. R. Power and M. S. Ferreira, Phys. Rev. B 83, 155432 (2011).

[69] R. Kubo, J. Phys. Soc. Jpn. 12, 570 (1957).

[70] A. Bastin, C. Lewiner, O. Betbeder-Matibet, and P. Nozieres, J. Phys. Chem. Solids 32, 1811 (1971).

[71] A. Crépieux and P. Bruno, Phys. Rev. B 64, 014416 (2001).

[72] P. Streda, J. Phys. C 15, L717 (1982).

[73] J. H. García, L. Covaci, and T. G. Rappoport, Phys. Rev. Lett. 114, 116602 (2015).

[74] J. H. Garcia and T. G. Rappoport, 2D Mater. 3, 024007 (2016).

[75] J. H. Garcia, A. W. Cummings, and S. Roche, Nano Lett. 17, 5078 (2017).

[76] Z. Fan, J. H. Garcia, A. W. Cummings, J. E. Barrios-Vargas, M. Panhans, A. Harju, F. Ortmann, and S. Roche, Phys. Rep. (2021), doi:10.1016/j.physrep.2020.12.001.

[77] A. Ferreira, T. G. Rappoport, M. A. Cazalilla, and A. H. Castro Neto, Phys. Rev. Lett. 112, 066601 (2014).

[78] M. Milletarì and A. Ferreira, Phys. Rev. B 94, 134202 (2016).

[79] V. Bonbien and A. Manchon, Phys. Rev. B 102, 085113 (2020).

[80] C. Caroli, R. Combescot, P. Nozieres, and D. Saint-James, J. Phys. C 4, 916 (1971).

[81] C. H. Lewenkopf and E. R. Mucciolo, J. Comput. Electron. 12, 203 (2013).

[82] A. Cresti, R. Farchioni, G. Grosso, and G. P. Parravicini, Phys. Rev. B 68, 075306 (2003).

[83] B. K. Nikolić, L. P. Zârbo, and S. Souma, Phys. Rev. B 73, 075303 (2006).

[84] S. R. Power, M. R. Thomsen, A.-P. Jauho, and T. G. Pedersen, Phys. Rev. B 96, 075425 (2017).

[85] M. Vila, J. H. Garcia, A. W. Cummings, S. R. Power, C. W. Groth, X. Waintal, and S. Roche, Phys. Rev. Lett. 124, 196602 (2020). 